\title{
Gene therapy for ocular diseases meditated by ultrasound and microbubbles (Review)
}

\author{
CAIFENG WAN, FENGHUA LI and HONGLI LI
}

Department of Ultrasound, Renji Hospital, School of Medicine, Shanghai Jiao Tong University, Shanghai 200127, P.R. China

Received July 29, 2014; Accepted June 3, 2015

DOI: $10.3892 / \mathrm{mmr} .2015 .4054$

\begin{abstract}
The eye is an ideal target organ for gene therapy as it is easily accessible and immune-privileged. With the increasing insight into the underlying molecular mechanisms of ocular diseases, gene therapy has been proposed as an effective approach. Successful gene therapy depends on efficient gene transfer to targeted cells to prove stable and prolonged gene expression with minimal toxicity. At present, the main hindrance regarding the clinical application of gene therapy is not the lack of an ideal gene, but rather the lack of a safe and efficient method to selectively deliver genes to target cells and tissues. Ultrasound-targeted microbubble destruction (UTMD), with the advantages of high safety, repetitive applicability and tissue targeting, has become a potential strategy for gene- and drug delivery. When gene-loaded microbubbles are injected, UTMD is able to enhance the transport of the gene to the targeted cells. High-amplitude oscillations of microbubbles act as cavitation nuclei which can effectively focus ultrasound energy, produce oscillations and disruptions that increase the permeability of the cell membrane and create transient pores in the cell membrane. Thereby, the efficiency of gene therapy can be significantly improved. The UTMD-mediated gene delivery system has been widely used in pre-clinical studies to enhance gene expression in a site-specific manner in a variety of organs. With reasonable application, the effects of sonoporation can be spatially and temporally controlled to improve localized tissue deposition of gene complexes for ocular gene therapy applications. In addition, appropriately powered, focused ultrasound combined with microbubbles can induce a reversible disruption of the blood-retinal barrier with no significant side effects. The present review discusses the current status of gene therapy of
\end{abstract}

Correspondence to: Professor Fenghua Li or Dr Hongli Li, Department of Ultrasound, Renji Hospital, School of Medicine, Shanghai Jiao Tong University, 1630 Dongfang Road, Shanghai 200127, P.R. China

E-mail: lihonglidoctor@sina.com

E-mail: lifenghuaprof@sina.com

Key words: ultrasound, microbubble, gene therapy, ocular ocular diseases as well as studies on gene therapy of ocular diseases meditated by UTMD.

\section{Contents \\ 1. Introduction \\ 2. Barriers for ocular gene therapy \\ 3. Current status of gene therapy of ocular disease \\ 4. Mechanisms of ultrasound contrast agent-mediated gene delivery \\ 5. Gene transfer mediated by UTMD in ocular disease \\ 6. Conclusion}

\section{Introduction}

Millions of people suffer from a variety of ocular diseases, several of which may lead to vision impairment and even complete blindness $(1,2)$. In spite of recent progress in diagnosis and treatment, numerous ocular diseases remain the leading cause of blindness in adults. At present, there is no satisfactory treatment available for these disorders; hence, it is imperative to develop more effective treatments as well as preventive methods. Gene therapy, which can be defined as the delivery of nucleic acids into targeted cells to exert a therapeutic effect, is a promising technology for treating currently incurable diseases, including malignant tumors and debilitating genetic disorders. The eye is an immune-privileged organ and has structural and accessibility properties that make it an ideal target organ for gene therapies. With increasing insight into the molecular mechanisms of ocular diseases, gene therapy has been proposed as a promising therapeutic tool for ocular diseases $(3,4)$.

Gene vectors are among the most important factors in gene therapy. Successful gene therapy depends on efficient gene transfer to targeted cells to warrant stable and prolonged gene expression with minimal toxicity. Clinical applications of gene translation are currently hampered due to a lack of a safe, efficient and non-invasive means to selectively deliver genes to target cells. With the advances in preparation technology of microbubbles and innovations in ultrasound imaging, ultrasound is no longer confined to the detection of tissue perfusion, but gradually expands to specific molecular imaging and targeted therapies. In recent years, numerous studies have 
indicated that ultrasonic irradiation itself not only promotes gene transfection, and that ultrasound-mediated microbubble destruction (UTMD) can further enhance gene transfection efficiency in vitro and in vivo (3-8). UTMD-mediated gene delivery systems have been widely used in pre-clinical studies to enhance gene expression in a site-specific manner in a variety of organs and tissues (8-10). In the sphere of ophthalmology, the application of the UTMD-meditated gene therapy has also been proved to be efficient (11-13). The present article discussed the current status of gene therapy of ocular diseases and reviewed the progress in the delivery of genes to ocular by UTMD.

\section{Barriers for ocular gene therapy}

Effective delivery of bioactive molecules to regions of pathology is dependent on numerous factors that are often difficult to control. The major challenge is the site-specific delivery of the payload to the target tissues and its subsequent transport across the endothelial barrier. The eye's unique anatomy and its physiological and anatomical barriers can limit effective gene delivery into the eye. In ocular gene therapy, one of the major challenges is to overcome intracellular and extracellular barriers. Various barriers present at the anterior and posterior segments of the eye restrict the entry of the gene material.

The cornea, which is an avascular tissue, is a good target tissue to evaluate gene therapy owing to its simple histological structure, immune-privileged nature and easy accessibility. Its primarily consists of external stratified epithelium, a thick collagenous stroma and a cuboidal monolayer of epithelial-like cells called endothelium (14). The stratified epithelium is composed of six to seven layers of stratified epithelial cells with tight junctions, and the tight junctions create a major barrier to topical gene delivery. Kamata et al (15) demonstrated that the tight junction of epithelial and Bowman's membrane constrained viral invasion. The tight junctions are the main barriers of the anterior segment of the eye regarding the transport of genes. The collagenous stroma is mainly composed of the predominant stromal cells and an extracellular matrix. It is separated from the corneal epithelium by a condensed collagenous layer, Bowman's membrane, and from the endothelium by a thin acellular layer, Descemet's membrane (14). Klausner et al (16) reported that administration of viral vectors via the epithelium or endothelium does not result in efficient transduction of the stromal keratocytes. The endothelium is the innermost monolayer, forming a leaky barrier positioned between the stroma and aqueous humour. Kamata et al (15) also reported that gene expression was restricted to endothelial cells after the injection of a viral vector into the anterior chamber of a mouse eye.

With regard to ocular gene therapy, target cells are often located in the neuroretina or the retinal pigment epithelium (RPE). The most convenient way of therapeutic gene delivery to them would be topical application; however, due to the limited diffusion of the gene particles through the sclera, the delivery efficiency is low. For systemic administration, the retina and vitreous are inaccessible due to the tight blood-retinal barrier (BRB). Topical application and systemic administration are thus less suitable for the delivery of gene material to the retina and RPE. Therefore, in most ocular gene therapy trials, sub-retinal or intravitreal injection are possible routes of administration for gene complexes. Although sub-retinal injection has shown encouraging results in certain studies, this invasive method is not always the first choice. Intravitreal and topical delivery of liposomes to the eye have been reported $(17,18)$; however, they have yielded low transfection efficiency in the retina and RPE. Dalkara et al (19) reported that the inner limiting membrane and BRB severely limit the passage of adeno-associated virus (AAV) after intravitreal delivery. Intravitreal delivery is an invasive procedure with risk of retinal detachment, hemorrhage, endophthalmitis and glaucoma $(20,21)$. Following intravitreal injection, it is difficult for a gene complex to diffuse through the vitreous. If the target is the RPE, the neural retina is thought to be another barrier. Peeters et al (22) and Du et al (23) found that the neural retina is a significant barrier for the delivery of non-viral gene complexes to the RPE.

Vitreous humour is a gel-like material that consists of collagen, hyaluronan, and proteoglycans containing chondroitin sulfate and heparan sulfate (24). Three-dimensional networks of the collagen fibrils are cross-like with proteoglycan filaments that contain negatively charged glycosaminoglycans (GAGs) (24). Size and charge are thought to be the main factors that limit the movement of the gene carriers in the vitreous humour. The negatively charged GAGs present in the vitreous humour may bind to the gene complexes, and gene materials may therefore become stuck to the gel-like materials in the vitreous humour (25). Considering its structure and composition, the vitreous humour may decrease the gene transfer efficiency. Du et al (23) demonstrated that the biopolymer network in the vitreous humour decreased the delivery efficiency of nanoparticles loaded with small interfering (si)RNA to RPE-J cells in vivo. Retinal gene delivery is a challenging area in the field of ocular gene delivery. With regard to gene delivery to the posterior segments of the eye, the BRB, vitreous and neural retina are likely to be the main barriers.

A series of biological and physiological barriers associated with almost all aspects of cellular biology are required to be overcome in order to achieve efficient gene delivery. Firstly, when systemically injected, the gene vectors are required to pass through the endothelial barrier of the capillary wall. The gene complexes face the threat of being rapidly degraded by the DNAse in the serum or the immune system prior to reaching the target cells. Nishikawa and Huang (26) demonstrated that the no-viral DNA vectors are often rapidly cleared from the circulation by mononuclear phagocyte systems. Manickan et al (27) demonstrated that viral vectors are rapidly cleared by hepatic Kuppfer cells, which may result in high deposition in the liver and even liver toxicity. Secondly, it must be avoided that gene complexes are entrapped into the endosome or the lysosome, where they are degraded. Thirdly, the gene complexes are required to penetrate the nuclear membrane to achieve the goal of gene expression for successful gene therapy. In summary, a variety of intracellular and extracellular barriers are required to be overcome for efficient gene delivery.

\section{Current status of gene therapy of ocular disease}

As gene therapy begins to produce its first clinical successes, interest in ocular gene therapy has grown owing to the favor- 
able safety and efficacy characteristics of the eye as a target organ for gene delivery. The basic technology of gene delivery systems is divided into two categories: Viral vector-mediated methods and a non-viral vector-mediated methods. Over the last decades, numerous viral and non-viral vector-mediated gene transfer methods have been tested in a large number of animal models of ocular diseases.

Viral vectors commonly used for ocular gene transfer are adenoviral (28), adeno-associated viral (AAV) (29) and lentiviral vectors (30). Viral systems can provide highly efficient delivery into cells with sustained expression. Recently, Igarashi et al (28) showed that vascular endothelial growth factor (VEGF)-targeted siRNA can be expressed across the retina and that long-term suppression of choroidal neovascularization (CNV) is possible through the use of stable AAV2/8-mediated VEGF siRNA expression. AAV2/8-mediated VEGF siRNA expression may be a feasible method to manage CNV in conditions such as age-associated macular degeneration. Huang et al (29) conducted a study to evaluate whether AAV-mediated overexpression of growth-associated protein-43 (GAP-43) has protective or deleterious effects on retinal ganglion cell (RGC) survival in laser-induced chronic intraocular pressure (IOP) elevation injury. The study showed that AAV mediated the overexpression of the axonal growth-associated protein GAP-43 in RGCs and severely aggravated RGC death in experimental glaucomatous injury. At present, the main disadvantage of viral systems is their potential for uncontrollable and insertional mutagenesis (31). Viral vectors evoke immune responses independent of the transgene constructs used, vector dose or vector preparation, which limits repetitive regimens $(32,33)$. Furthermore, the transduction of certain viral vectors occurs with relatively low efficiency, which limits its therapeutic effects (34). The potential dangers of viral vectors may hamper their further development for ocular gene therapy in humans (35). These limitations have prompted a requirement to develop non-viral delivery systems with high biosafety and low cytotoxicity.

In the last decade, the development of non-viral methods for ocular gene therapy has made great progress in cell lines and animal models. Non-viral delivery approaches are constituted by chemical methods (mainly involving cationic lipids, polymers or nanoparticles) and physical methods (mainly involving administration by gene gun, electroporation, iontophoresis or microinjection) $(36,37)$. Approaches based on utilization of non-viral vectors are easily available, cost-effective and do not evoke any antigen-specific immune and inflammatory responses after ocular administration (38). The emergence of nanotechnology may have a profound effect on ocular biomedical applications, particularly the delivery of drugs to the posterior of the eye via nanocarriers $(39,40)$. Jayaraman et al (41) synthesized a nanoformulation consisting of a water-soluble chitosan conjugated with a peptide (serine-threonine-tyrosine) as a potential carrier for retinal delivery to treat age-associated macular degeneration (AMD). In this study, the conjugated nanochitosan peptide showed evidence of tyrosine kinase activity as indicated by fluorescent signals under the confocal microscope, while nanochitosan or peptide alone did not show such activity. Zhou et al (42) designed a study for investigating the downregulation of mRNA expression of VEGF by triamcinolone acetonide acetate (TAA)-loaded chitosan nanoparticles in human retinal pigment epithelial cells. The study demonstrated that TAA/loaded deoxycholic acid (DA)-modified chitosan nanoparticles had a downregulating effect on VEGF mRNA expression in human retinal pigment epithelial cells with low cytotoxicity; these are beneficial characteristics suggesting the suitability of these chitosan-derived nanoparticles to be developed into therapeutics for diabetic retinopathy. Although non-viral vector-meditated gene transfer efficiency has improved over the past decade, it remains relatively low and the expression duration of the transgene is relatively short (43). Regarding physical methods, their inherent risks may outweigh their benefits, rendering them inappropriate for ocular gene transfer, and the invasive nature of these methods reduces patient compliance for effective therapy (44). The major obstacle in the clinical application of gene therapy is not the lack of ideal genes, but rather the lack of a clinically safe and efficient gene transfer method (45). Therefore, it is necessary to develop effective and specific ocular gene delivery systems.

UTMD-mediated gene delivery systems hold promise to fulfill this void, particularly with the wide use of ultrasound contrast agents in clinical diagnostic imaging. UTMD-meditated gene delivery, with the advantages of low toxicity, a high safety profile, repetitive applicability and specific tissue targeting, provides a novel method for gene therapy $(46,47)$. Lin et al $(48)$ reported that focused ultrasound with microbubbles was able to effectively transfer nanoparticles into mouse tumors through altering the permeability properties of the vasculature and cell membrane. It was reported that the delivery of the TFPI-2 gene using SonoVue was able to suppress thrombosis and arterial restenosis, providing a potential gene therapy approach for atherosclerosis (49). With the extensive research on ultrasound contrast agents in gene transfer and gene therapy, an increasing number of researchers are beginning to introduce the application of UTMD for ocular gene transfection.

\section{Mechanisms of ultrasound contrast agent-mediated gene delivery}

Microbubble contrast agents, although typically used to enhance ultrasound contrast for imaging, are increasingly gaining attention due to their ability to directly deliver various classes of bioactive substances to a number of tissue types, and becoming increasingly popular for targeted gene and drug delivery, as well as the monitoring thereof. UTMD has evolved as a promising system for non-invasive, target-specific gene delivery. The low toxicity and simplicity of its in vivo application make this technology particularly attractive. Ultrasound contrast agents are gas-filled spheres remaining completely intravascular when systemically injected. Microbubbles as cavitation nuclei are able to volumetrically expand and contract in response to compression and rarefaction phases of ultrasound waves. When the acoustic pressure reaches a certain threshold, microbubbles violently collapse and cause a series of biological effects. The physical response of microbubbles can mechanically perturb the integrity of blood vessel walls and cell membranes, thus increasing their permeability to therapeutic agents, which can thereby penetrate into the cells (50). Sirsi and Borden (50) categorized the mechanisms 


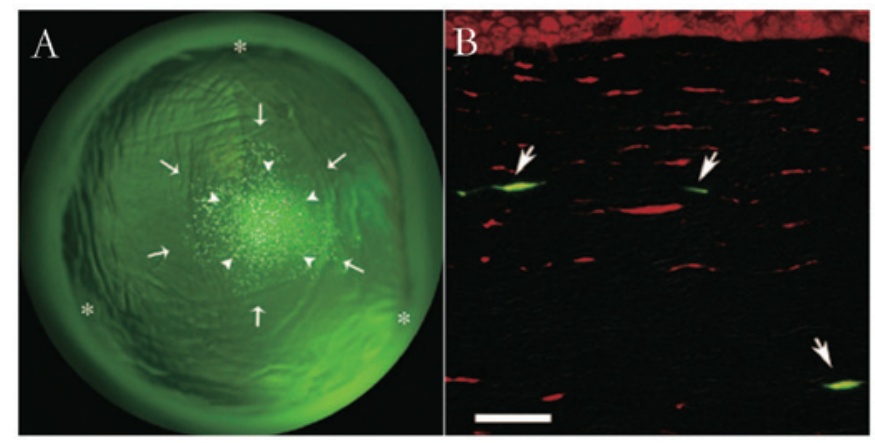

Figure 1. Fluorescence micrographs of rabbit cornea after treatment with US and MB. (A) Asterisks indicate the corneal margin. Arrows indicate where plasmid and MBs were injected. Arrowheads indicate exactly where the US probe was placed. GFP-positive cells were observed exclusively where US was applied. (B) Fluorescence microscopic examination showed that GFP was present in spindle-shaped cells in the targeted regions of the corneal stroma. The image was taken from Sonoda et al (9) (scale bar, $10 \mu \mathrm{m}$ ). US, ultrasound; MB, microbubble; GFP, green fluorescence protein.

of the alternation of the vascular permeability by microbubble cavitation into three different classes: i) Creation of transient pores in vascular endothelial cells that allow intracellular macromolecule uptake; ii) disruption of vascular endothelial integrity; iii) stimulation of endocytotic cellular uptake.

Due to shock waves and jetting during microbubble collapse, the inertial cavitation of microbubbles can cause transient membrane ruptures. This phenomenon termed as 'microbubble sonoporation', was thought to be the primary mechanism of intracellular gene delivery (51). Studies have reported that microbubbles enhanced gene delivery efficiency by lowering the cavitation threshold and enhance cavitation erosion (52), and the 'spillover space' on the cell membrane after the microbubble cavitation lasted for $24 \mathrm{~h}$, which is sufficient for gene entry and expression (53). Zhou et al (54) reported that a single microbubble was able to generate transient pores of a size proportional to the proximity of the cavitation event to the membrane, and the membranes returned to normal within 20 sec. UTMD-meditated pore formation was a highly effective and controllable approach that transiently disrupted the membrane integrity to enhance its permeability to circulating agents. Volumetric expansion of the microbubble in the ultrasound field can facilitate bubble-vessel interaction, while microbubble oscillation exerts a longitudinal strain on blood vessels, and can partially embed them in the endothelium and continue to oscillate, which may alter vascular permeability (55). Hauser et al (56) studied the effects of stable microbubble cavitation on endocytotic activity in cultured cells, and demonstrated that stable cavitation of microbubbles increased the number of clatherin-coated pits and endocytotic vesicles. The study also demonstrated that stable cavitation of microbubbles can increase endocytotic activity of cultured cells under low-intensity ultrasound (56). To date, the exact mechanisms governing the enhancement of UTMD-mediated gene delivery in vivo have remained to be fully elucidated; however, acoustic cavitation is thought to be a major contributor. The biological effects of ultrasound, including microstreaming and other convective phenomena, are also thought to contribute to the enhancement of gene delivery.

\section{Gene transfer mediated by UTMD in ocular disease}

UTMD-meditated gene delivery provides a novel method for gene therapy. In recent years, numerous in vitro and in vivo studies have conformed that ultrasound with microbubbles significantly enhanced gene transfection efficiency. UTMD is therefore emerging as a powerful tool for the treatment of ocular diseases.

UTMD-meditated gene transfer to the cornea. The cornea is an ideal tissue for studies on gene transfer, as it is transparent and avascular. Sonoda et al (9) investigated the practical efficacy and safety of ultrasound plus microbubble-mediated gene transfer to cornea in vitro and in vivo. While treatment with DNA alone did not lead to any gene transfer into the cultured corneal epithelial cell line RC-1, ultrasound slightly enhanced gene transfer, and ultrasound plus microbubbles significantly increased the gene transfer efficiency. In the in vivo study, ultrasound plus microbubbles markedly increased gene transfer efficiency without any apparent tissue damage. Green fluorescence protein (GFP)-positive cells were observed exclusively where ultrasound had been applied and GFP was mainly present in spindle-shaped cells in the targeted regions of the corneal stroma (Fig. 1) (10). Yamashita et al (45) used a novel bubble liposome (BL) composed of a polyethylenglycol (PEG)-modified liposome containing perfluoropropane gas, with ultrasound to transport GFP into rabbit RC-1 cells in vitro and conjunctiva in vivo. The study showed that $\mathrm{BL}$ with US effectively transferred genes into cultured corneal epithelial cells and rat sub-conjunctival tissue without causing any apparently adverse effects. Diffuse fluorescence-positive granules were present in sub-conjunctival tissues and no tissue damage was observed histologically (Fig. 2).

UTMD-meditated gene transfer to the retina. Gene transfer provides a novel approach for the treatment of retinal diseases. Li et al (57) demonstrated that UTMD was able to safely and effectively deliver plasmids into RGCs in vitro. Under the optimum parameters, the average transfection rate of p enhanced (E)GFP-N1 with UTMD was $25 \%$. Compared with the ultrasound plus plasmid group, the number of transfected cells increased by 28 -fold. Another study reported that UTMD-mediated gene transfer of pigment epithelium-derived factor (PEDF) into retina and chorioids of rats inhibited the development of CNV (58). The study also demonstrated that in the short term ( 7 and 14 days after transfection), the transfection efficiency mediated by UTMD was not different from that achieved by liposome-based gene transfer. However, in the long term (28 days after transfection), the transfection efficiency by UTMD was significantly higher as compared with that of the liposome approach. The shock wave of UTMD promoted the delivery of the plasmid into the cell nucleus, which may partly be explained by the induction of tight binding of the target plasmid to the cell's endogenous DNA. UTMD therefore presents a solution for local gene transfection and reduces the amount of plasmid required. Sonoda et al (13) used a miniature ultrasound transducer to evaluate the efficacy of intravitreal ultrasound (SonoPore 4000) irradiation for selective GFP plasmid transfer into the rabbit retina. The ultrasound probe, as small as a 19-gauge needle, was inserted into the vitreous 

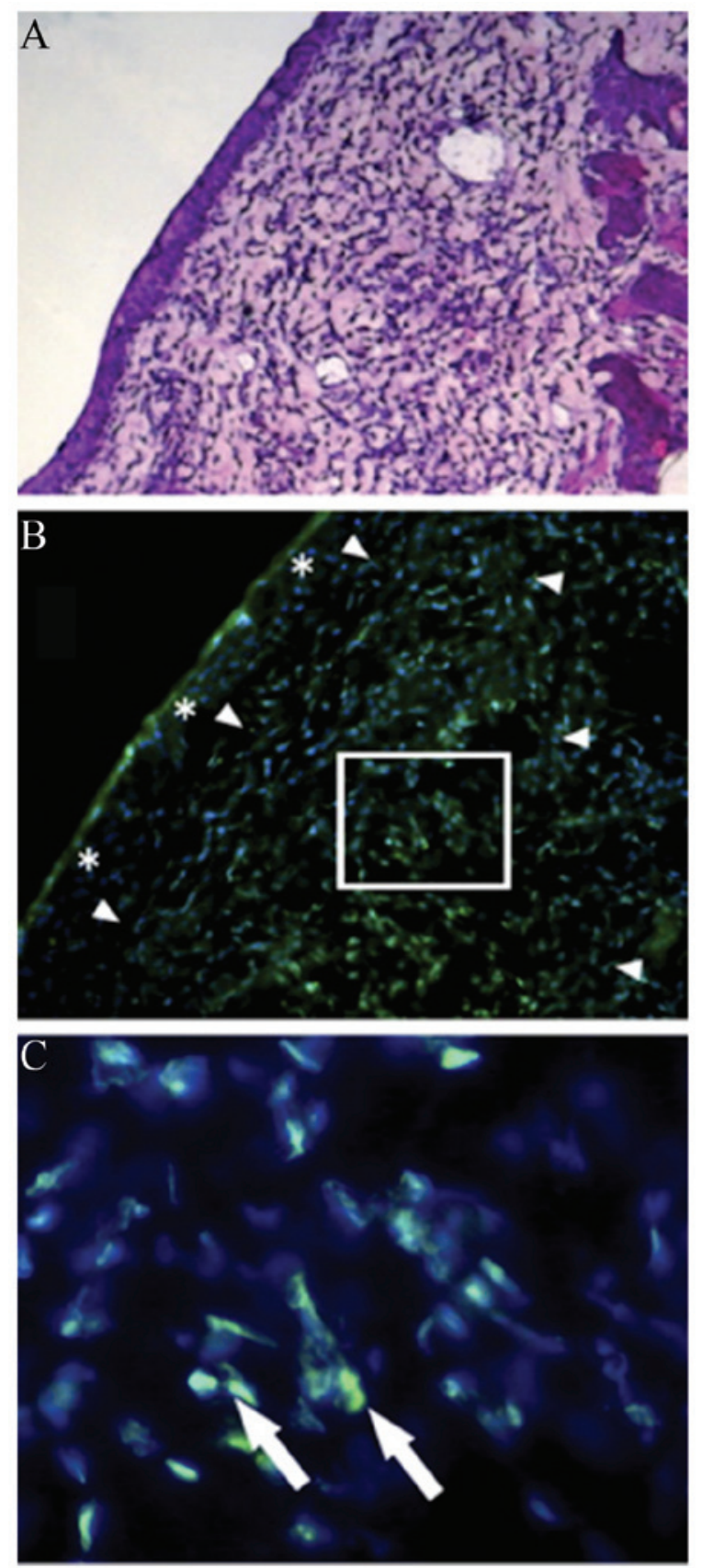

Figure 2. Microscopic images of rat conjunctiva following exposure to bubble liposomes and US. (A) Light microscopic image of hematoxylin- and eosin-stained tissue (magnification, x20). (B) Fluorescent microscopic examination showed that GFP was present in spindle- to round-shaped cells beneath the conjunctival epithelium of the area exposed to US with no obvious tissue damage (magnification, x20). Arrowheads indicate conjunctival stroma and asterisks indicate epithelium of conjunctiva. (C) Enlarged section of B marked by white square (magnification, $\mathrm{x} 40$ ). GFP was mainly located in the cytoplasm of these cells (arrow). Image taken from Yamashita et al (45). US, ultrasound GFP, green fluorescence protein.

cavity through a scleral incision. The gene-transfer efficiency was quantified by counting the number of GFP-positive cells. The study demonstrated that the retinas that received plasmid with BL and ultrasound showed a significant increase in the number of GFP-positive cells without any apparent tissue damage (Fig. 3) (13). GFP-positive cells were observed exclusively in the area that was exposed to ultrasound, and no GFP-positive cells were observed in the control eyes that were not treated with ultrasound. These results indicated that gene delivery to the retina using intravitreal ultrasound exposure is more selective than the transcorneal method.

RNA interference is a promising biological strategy for the treatment of diseases; however, its instability and poor cellular uptake have limited its application (59). To overcome such limitations, an siRNA delivery method based on the combined use of nanoparticles with ultrasound and/or microbubbles was used in a number of studies. Du et al (23) designed a study to investigate the efficacy and safety of ultrasound and/or microbubble-enhanced delivery of monomethoxypoly (ethylene glycol)-poly(lactic-co-glycolic acid)-poly L-lysine (mPEG-PLGA-PLL) nanoparticles loaded with platelet-derived growth factor BB (PDGF-BB) siRNA into rat RPE-J cells. The results showed that ultrasound and/or microbubbles were able to be used safely to enhance the delivery of nanoparticles loaded with siRNA to rat RPE-J cells, and this approach downregulated the mRNA and protein expression of PDGF-BB with enhanced efficiency. However, the combination of ultrasound and microbubbles under the optimal conditions did not further increase the cellular uptake of nanoparticles compared to that achieved with either ultrasound or microbubbles alone. In vitro, the rat RPE-J cells are fragile and vulnerable, and the bio-effects of UTMD may have been too aggressive to further increase the delivery of nanoparticles, which may explain the observations of the study.

AAV vectors possess a number of advantages over other vectors, which render them suitable for transfection studies, in particular their ability to transfect cells in a stable and long-term manner and their relative lack of pathogenicity (1). Viral vectors are usually delivered systemically, which may lead to anti-viral immune responses of the host. Due to the immunoreaction and the limits of the endothelial barrier, the transduction of these viral vectors occurs with relatively low efficiency, which limits its therapeutic effects. Increasing viral vector transduction may produce improved therapeutic effects (34). UTMD-meditated local gene therapy has the potential not only for plasmid-DNA transfer, but also virus-mediated gene transfer. A study demonstrated that a microbubble can load and protect an adenoviral vector, and that the delivery system comprising the vector incorporated into the microbubble was able to improve site-specific targeting of GFP (60). The study also proved that the microbubble was able to reduce the degradation rate of the viral vectors after intravenous injection. In analogy with this, Geers et al (61) found that UTMD can specifically and effectively increase recombinant (r)AAV-mediated gene delivery. Li et al (62) demonstrated that UTMD enhanced rAAV2 transfer efficiency into less permissive hRCC cells by two- to three-fold without decreasing cell viability. Polymerase chain reaction analysis showed that with the use of UTMD, a more than nine-fold enhancement of rAAV2 genomic DNA copies was achieved compared with that using rAAV2 alone in vitro, and a more than two-fold enhancement in vivo. In the in vivo study, UTMD not only amplified rAAV2 transduction, but also inhibited tumor growth.

Compared to other viral vectors that have recently been investigated, rAAV has the advantages of low immunogenicity and stable long-term transgene expression (63), which has been widely studied in retinal diseases. Ultrasound-meditated microbubble destruction was able to enhance $\mathrm{rAAV}$-mediated 

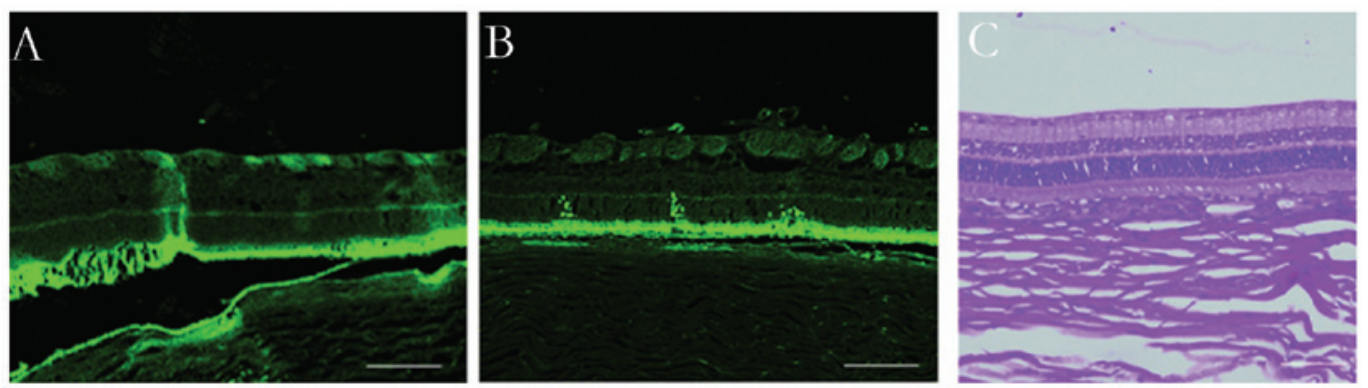

Figure 3. Fluorescence and H\&E staining images of the rabbit fundus oculi. (A) In the plasmid + BL without US group, no GFP-positive cells were observed. (B) In the plasmid + BL + US group, GFP-positive cells were observed exclusively in the area exposed to US and mainly located in the outer nuclear layer. (C) H\&E staining showed no evident tissue damage after exposure of the intravitreal retina to BL with US. Scale bar, $100 \mu \mathrm{m}$. Image taken from Sonoda et al (13). H\&E, hematoxylin and eosin; US, ultrasound; BL, bubble liposome; GFP, green fluorescence protein.


Figure 4. Distribution of EGFP-positive cells in tissue-stretched preparation. The number of transfected cells in (A) the AAV and microbubble with ultrasound group was higher than that in (B) the AAV and normal saline group (magnification, x100). EGFP expression was mainly shown in (C) retinal pigment epithelial cells and (D) neural retina cells, respectively (magnification, x 400). Image taken from Li et al (4). EGFP, enhanced green fluorescence protein; AAV, adeno-associated virus.

gene delivery into retina cells $(11,12)$. Li et al (11) reported that UTMD enhanced rAAV2 transfection efficiency in human RPE cells in vitro and in Wistar rat retina in vivo. In this study, UTMD induced rAAV2-mediated EGFP expression earlier after injection and substantially increased gene expression prior to the peak (35 days) with no evident tissue damage. Fluorescence microscopic analysis of a tissue-stretched preparation showed that the number of EGFP-positive cells in the group treated with AAV, microbubbles and ultrasound was higher than that in the AAV and normal saline groups, and that EGFP expression mainly appeared in the layer of RPE cells and neural retina (Fig. 4). Recently, a study using mouse models of proliferative vitreoretinopathy demonstrated that UTMD produced a therapeutic effect by facilitating the insertion of rAAV2-conjugated genes into tumors (64). Xie et al (12) investigated the efficiency and safety of UTMD-mediated delivery of rAAV2-EGFP into RGCs of rats and demonstrated that EGFP expression in the group treated with rAAV2-EGFP, ultrasound and microbubbles was the highest, and that the number of transfected RGCs was the largest compared to that in the other groups. No obvious damage was observed by histopathological analysis. Zheng et al (65) investigated the feasibility of UTMD-enhanced rAAV or plasmid-mediated transfection into the human RPE cell line ARPE-19. The result showed that the transfection efficiency of rAAV and plasmid in ARPE-19 cells was enhanced by UTMD without any adverse effects on cell viability (Fig. 5). The transfection efficiency of rAAV was higher than that of plasmid. UTMD-enhanced rAAV-mediated transfection was therefore thought to be an appropriate method for retinal gene therapy.

UTMD combined with viral vectors offers numerous benefits: First, under ultrasound irradiation, microbubbles 

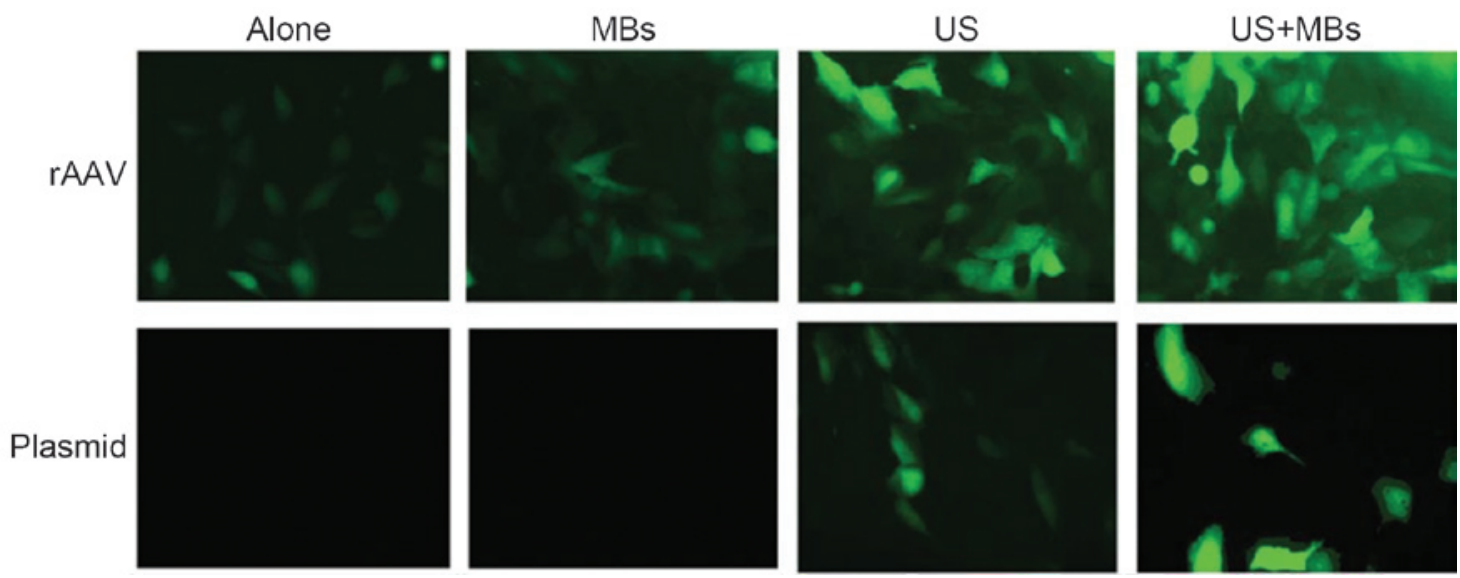

Figure 5. Transfection efficiency of rAAV and P in ARPE-19 cells alone, with MB, with US and with ultrasound-targeted MB destruction. No improvement in transgene expression was observed in the rAAV + MB and P + MB groups. Compared with the rAAV and P alone groups, the rAAV + US and P + US groups showed a weak but non-significant tendency to improve transgene expression. The ratio of enhanced green fluorescence protein-positive cells was significantly higher in the rAAV + US + MB and P + US + MB groups compared to that in the US alone group (magnification, x400). Image taken from Zheng et al (65). rAAV, recombinant adeno-associated virus; $\mathrm{P}$, plasmid; US, ultrasound; $\mathrm{MB}$, microbubble.

Time after exposure

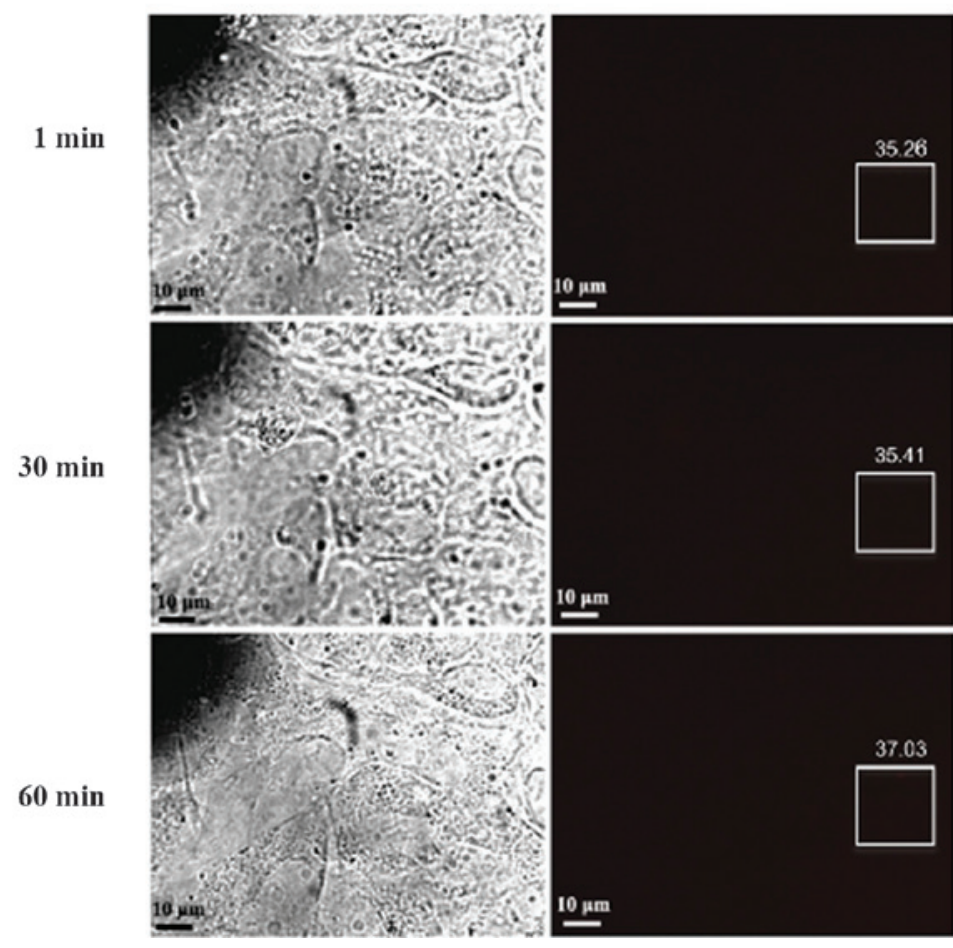

Doxorubicin with US + MB

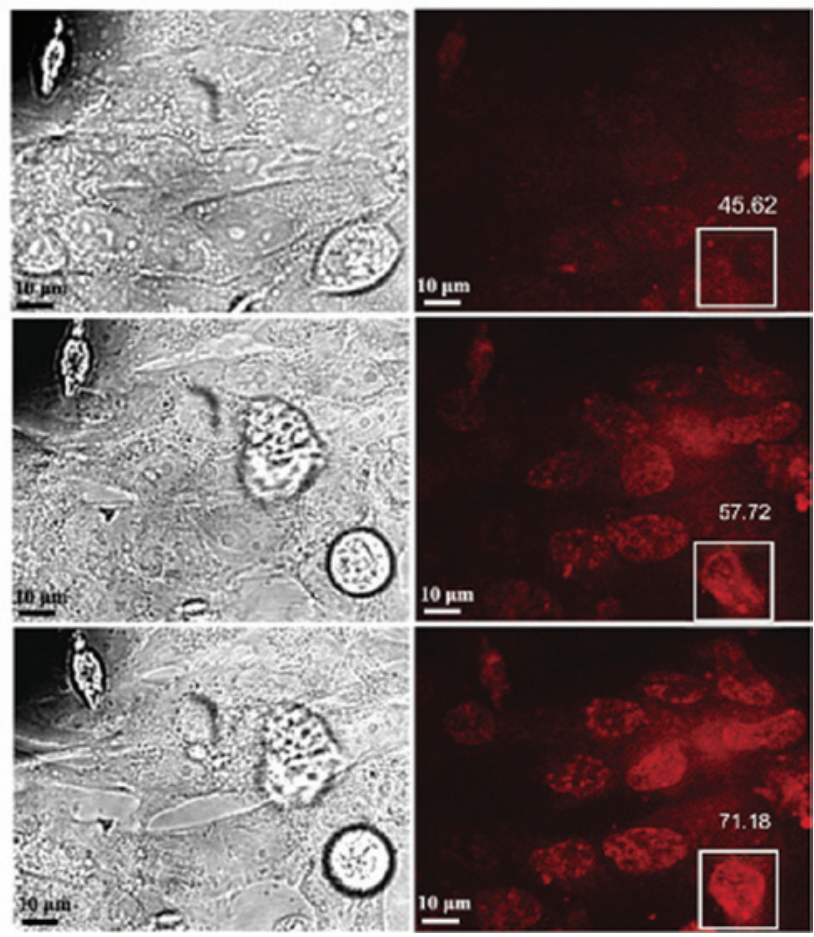

Figure 6. Intracellular fluorescent microscopic images of cells treated with doxorubicin alone or with doxorubicin + US + MB. As early as 1 min after sonoporation, cells treated with doxorubicin + US + MB showed increased intracellular fluorescence compared with cells exposed to doxorubicin alone ( 35.26 vs. 45.62). This effect increased further at $60 \mathrm{~min}$, where the mean intensity of fluorescence was 37.03 in control cells versus 71.18 in cells treated with doxorubicin + US + MB. Compared with the early fluorescence observed in cells exposed to US + MB, cells treated with doxorubicin alone showed only trace intracellular fluorescence at $60 \mathrm{~min}$. Boxes represent ROIs for measuring levels of fluorescence, and values indicate mean intensity of fluorescence within the ROI. Scale bars, $10 \mu \mathrm{m}$. Image taken from Lee et al (71). ROI, region of interest; US, ultrasound; MB, microbubble.

may improve the site-specific release of the viral vector (66). Second, UTMD can mechanically enhance the permeability of the blood vessel walls and cell membranes, and thus improve the transfer efficiency of the viral vector; and finally, the ultrasound contrast agent can simultaneously impose restrictions on the immune response to the viruses, thus allowing for intravascular administration and repetitive injection (60).
Jin et al (67) showed that UTMD stimulated the formation of clathrin-coated pits (CPs) as well as uncoated pits (nCPs), and facilitated the uptake of viral particles into the cytoplasm and nucleus for long periods of time, mainly by stimulating endocytosis. The combination of UTMD with viral vectors may represent a novel gene delivery system with high specificity and low invasiveness for patients with retinal diseases. 

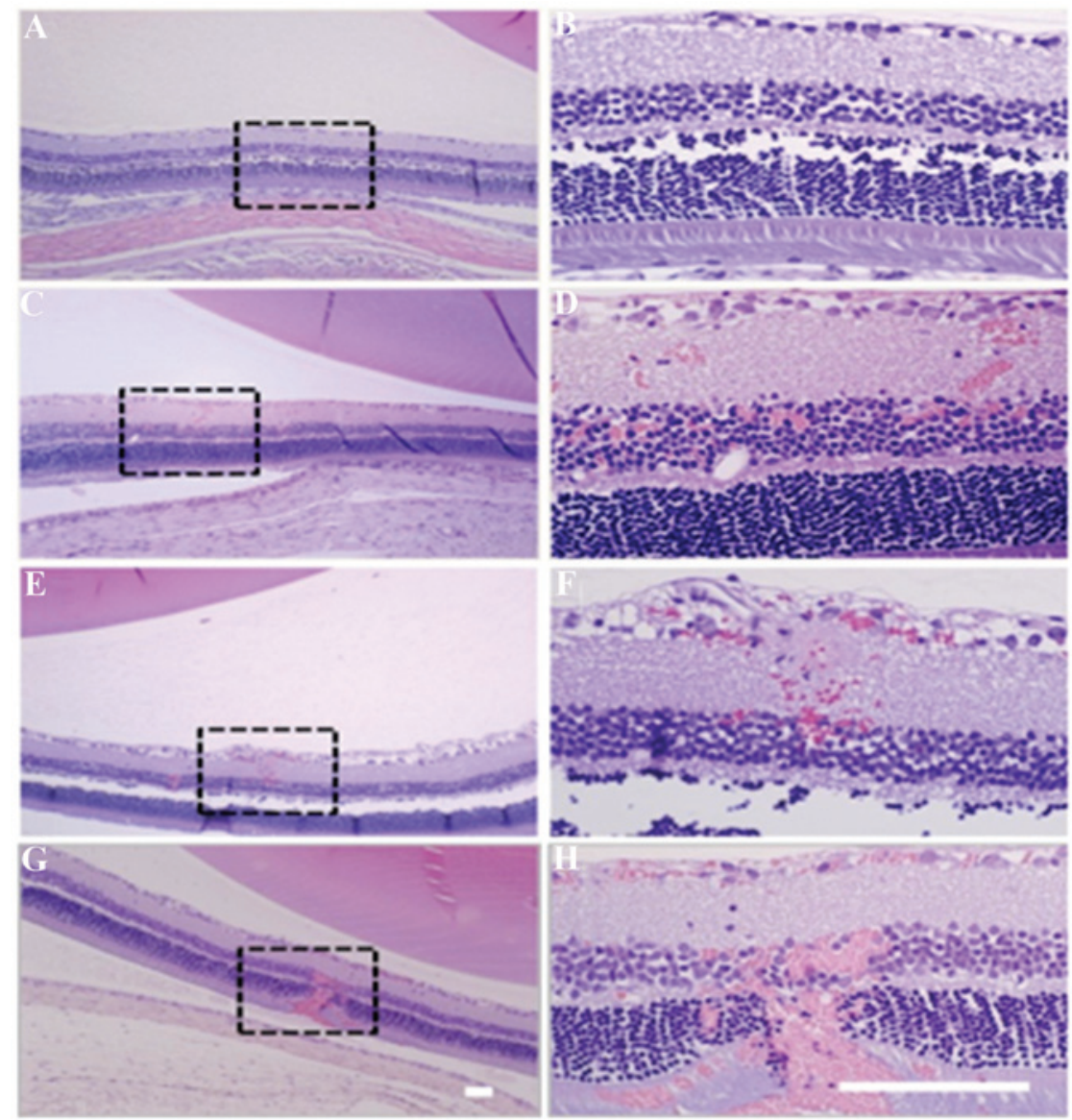

Figure 7. Micrographs of hematoxylin- and eosin-stained sections from each experimental group. Images on the right are high-magnification views of the boxed regions shown on the left. (A and B) Control eye without sonication; (C and D) $0.81 \mathrm{MPa}$; (E and F) $0.88 \mathrm{MPa}$; (G and H) $1.1 \mathrm{MPa}$. At 0.81 and $0.88 \mathrm{MPa}$, the retina in the sonicated region appeared to be generally unaffected except for a small number of small clusters of extravasated erythrocytes in the nuclear layers of the retina. More extensive damage was observed after sonication at 1.1 MPa. Scale bars, $100 \mathrm{~mm}$. Image taken from Park et al (76).

UTMD-meditated drug or gene transfer to retinoblastoma. In recent years, with the developments in molecular biology, gene therapy has been gaining importance in cancer therapy. Retinoblastoma (RB) is the most common malignant intraocular tumor in children. In spite of advances in enucleation and conservative treatments, there has been no improvement in the five-year survival rate in children. The treatment of retinoblastoma has been increasingly focused on localizing the therapy to the eye. UTMD-meditated drug or gene delivery to ocular tumors is regarded to be a non-invasive gene transfer technology and provides a novel means of gene therapy for retinal disease.

Microbubble destruction by ultrasound exposure generates microstreams or microjets that create shear stress on cells and open transient pores in cell membranes, which has the capability of transiently enhancing cell membrane permeability (68). The use of ultrasound with diagnostic microbubbles in cancer treatment to increase the efficiency of chemotherapy through passive, localized delivery has been an emerging area of research. Numerous studies have demonstrated that optimized UTMD-mediated therapy has the potential to improve cancer response to therapy via increased localized drug uptake and targeted therapeutics, which may lead to a lowering of chemotherapeutic drug dosages and systemic toxicity $(69,70)$. Lee et al (71) proved that using low-intensity $\left(0.3 \mathrm{~W} / \mathrm{cm}^{2}\right)$ and low-frequency $(1 \mathrm{MHz})$ ultrasound with microbubbles for
$10 \mathrm{sec}$ enhanced the chemotherapeutic efficacy of doxorubicin against retinoblastoma Y79 cells in vitro. Cells exposed to ultrasound and microbubbles showed earlier and higher trace intracellular fluorescence than that of cells treated with doxorubicin alone (Fig. 6). There is a significant decrease in cell viability in cells treated with this method compared with cells treated with chemotherapy alone. To investigate the duration and underlying mechanism of increased permeability, the study used scanning electron microscopy to image cells exposed to ultrasound + microbubbles (for 10 or $60 \mathrm{sec}$ ). Pores were identified in cells exposed to ultrasound + microbubbles for $60 \mathrm{sec}$ but not in those exposed for $10 \mathrm{sec}$. However, in vitro fluorescence showed that doxorubicin uptake significantly increased immediately after exposure to ultrasound + microbubbles for $10 \mathrm{sec}$. These results suggested that the presence of physical pores may not be a pre-requisite for enhanced drug entry into the cells. It is possible that transient electrical changes, endocytosis or other unidentified mechanisms contributed to the enhanced drug uptake. UTMD may become a valuable adjuvant to chemotherapy of RB, whose treatment is often limited by challenges in drug delivery, and may lead to more effective chemotherapy treatments with less damage and side effects to ocular tissues.

Luo et al (46) explored the efficiency of wild-type53 (wtp53) plasmid transfection into Y79 RB cells and RB xenograft tumor tissue meditated by ultrasound with microbubbles, 

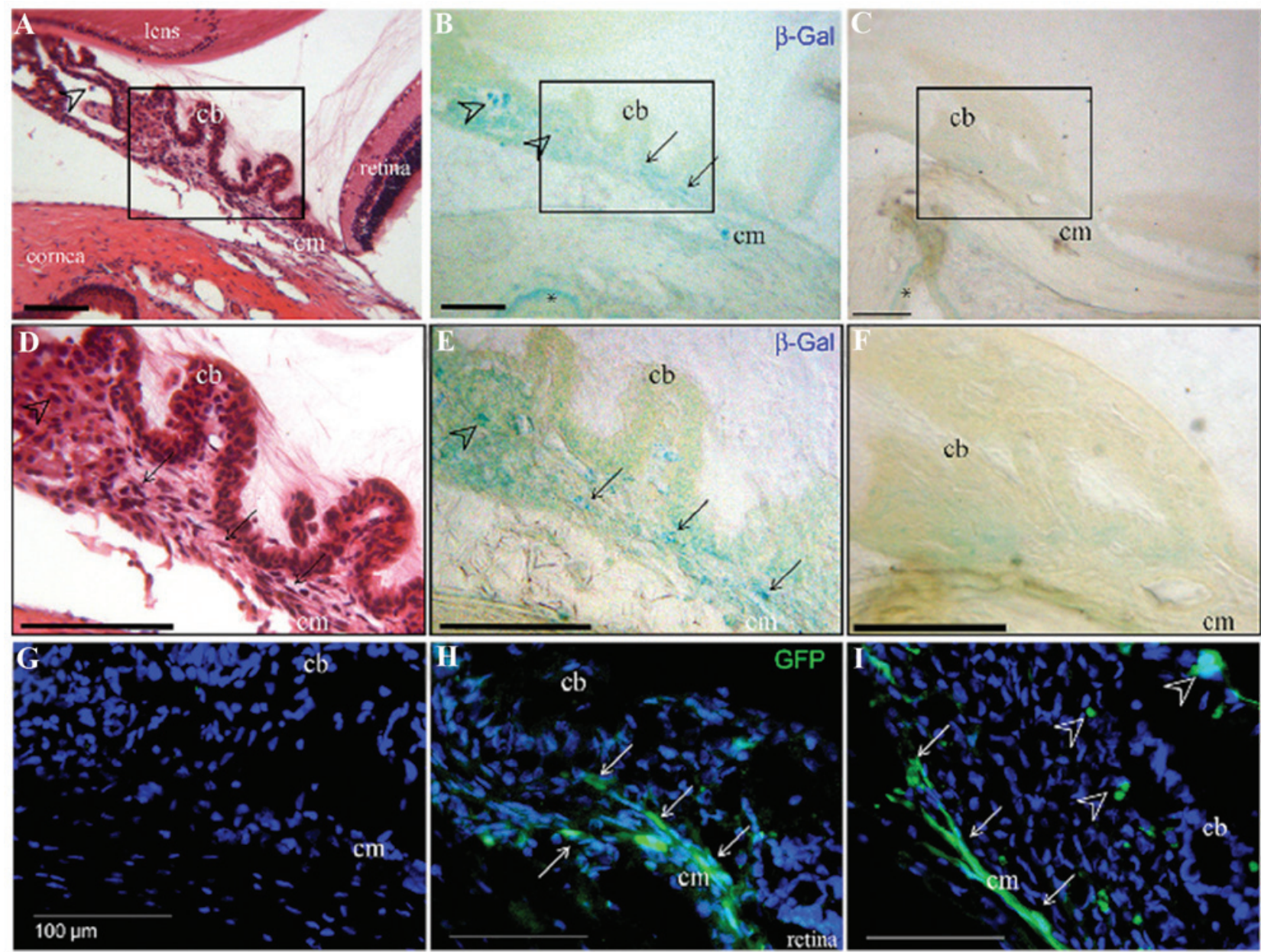

Figure 8. Localization of reporter proteins in the ciliary region. (A-E) Localization of $\beta$-galactosidase activity after injection of pVAX1-LacZ plasmid mixed with microbubbles (A, B, D and E) with sonoporation or (C and F) without sonoporation. (A and D) Staining with hematoxylin and eosin allowed localization of the ciliary muscle on slides directly mounted in glycerol/phosphate-buffered saline. (B and E) $\beta$-galactosidase activity was detected in muscle cells (arrows) and in a small number of cells around the ciliary body (arrow heads). ( $\mathrm{C}$ and F) No detectable $\beta$-galactosidase activity was present in the ciliary region without ultrasound application, except in the corneal epithelium, where staining was considered to be non-specific in all samples. (G-I) GFP was detected in (H and I) the fibers of the ciliary muscle (white arrows) and (I) in a small number of cells around the ciliary body (white arrowheads). No GFP-positive cells were observed in the control eyes (G). Scale bars, $100 \mathrm{~mm}$. Image taken from Kowalczuk et al (78). cb, ciliary body; cm, ciliary muscle; GFP, green fluorescence protein.

and demonstrated that the wtp53 gene was successfully transfected into solid tumors in the plasmid with microbubbles and ultrasound group. Flow cytometry showed that apoptosis was higher in the microbubbles and ultrasound group (25.58\%) compared with that in the plasmid with liposomes group $(19.50 \%)$ and the other two groups $(<10 \%)$. Another study used the same method to explore the transfection of the recombinant expression plasmid pEGFP-C1/RB94 into the human RB cell line HXO-Rb44 and examined the efficiency of RB94 in the inhibition of the growth of RB cells (72). The results showed that UTMD enhanced the transfection efficiency of RB94, which had an obvious impact on the growth inhibition of the RB cells. UTMD-meditated gene therapy may be a useful method for application in the gene therapy of RB.

UTMD-meditated reversible BRB disruption. The major challenge in delivering systemically administered substances to specific retinal locations is the existence of the BRB, which is formed by a complex tight junction of the retinal endothelial cells and the RPE. The BRB prevents most systemically administered drugs from reaching the retina. Almost $98 \%$ of clinically validated drugs are not able to cross the BRB (73). Multiple administration routes are currently used to deliver bioactive materials to the retina. However, topical, systemic and periocular approaches are limited by the BRB and other ocular barriers. Though sub-retinal and intravitreal injections can provide direct access for genes to the retina, these invasive methods are not the first choice for the treatment of the diseases of the eye due to the risk of the retinal detachment and hemorrhage (20). In addition, for certain chronic diseases requiring repeated administration, the risks multiply. Therefore, the development of minimally invasive and efficient methods that can bypass the BRB and enhance the delivery of therapeutic materials to the retina is desired.

A non-invasive, reversible and targeted technique that combines low-energy ultrasound bursts with a microbubble ultrasound contrast agent to temporarily induce blood-brain barrier (BBB) disruption was identified (74). The barrier can 
be restored without significant side effects, and the method was shown to improve therapeutic outcomes in animal disease models (75). In principle, similar techniques may be used to deliver drugs or genes to the retina. Using a rat model, Park et al (76) demonstrated that burst ultrasound together with an intravenously (i.v.) administered microbubble agent was able to induce transient increases of retinal vascular permeability for ocular drug delivery. For BRB disruption, 10-msec bursts were applied at $1 \mathrm{~Hz}$ for $60 \mathrm{sec}$ with different peak rarefactional pressure amplitudes $(0.81,0.88$ and $1.1 \mathrm{MPa})$. To evaluate $\mathrm{BRB}$ disruption, a magnetic resonance imaging contrast agent gadolinium diethylenetriaminepentaacetic acid (Gd-DTPA; Magnevist) was injected i.v. immediately after the last sonication, and serial T1-weighted magnetic resonance images were acquired at up to $30 \mathrm{~min}$. No signal enhancement was observed, suggesting that no Gd-DTPA leakage into the retina or vitreous humor was present in the non-sonicated animals. All of the animals that received ultrasound and microbubbles showed detectable signal enhancement. Though the maximum signal enhancement was greatest after sonication at $1.1 \mathrm{MPa}$, the retinal damage was severe (Fig. 7). Increased petechaie and retinitis were observed after sonication at 1.1 MPa. No significant retinal damage was identified by histological analysis at the two lower acoustic pressure amplitudes tested, and the barrier was found to be restored $3 \mathrm{~h}$ after sonication. The study demonstrated that appropriately powered focused ultrasound combined with microbubble induced a temporary and reversible disruption of the BRB in rats without any significant side effects. The BRB appeared to be restored within a few hours, which provided a suitable time-window for ocular pharmaceutical agent delivery while avoiding undesired effects that may result from long-term BRB disruption (76).

Ultrasound with microbubbles may offer a non-invasive, localized and repeatable means to reversibly disrupt the BRB for ocular substance delivery. To date, the mechanisms of the UTMD meditated BRB disruption have not been fully elucidated. The mechanical stimulation that induces a temporary widening of the tight junctions and the active transport may partly explain the beneficial effects of UTMD on gene delivery and drug uptake (77).

UTMD-meditated gene transfer to the ocular ciliary muscle. Kowalczuk et al (78) assessed the application of low- intensity ultrasound combined with commercial microbubbles to transfect the ciliary muscle of rat eyes. The ultrasound settings applied were as follows: $1 \mathrm{MHz}, 2 \mathrm{~W} / \mathrm{cm}^{2}$ and a $50 \%$ duty cycle of $2 \mathrm{~min}$. At 1 week, the ultrasound + microbubble treatment produced a significant increase in luminescence compared with that in the control eyes injected with plasmid only, with or without microbubbles. The reporter proteins were localized in the ciliary muscle as indicated by histochemical analysis (Fig. 8). At 1 month, all groups showed a significant decrease in luciferase activity. A rise in lens and ciliary muscle temperature was detected during the procedure; however, this did not result in any observable damage at 1 and 8 days. This study demonstrated that the ocular ciliary muscle can be targeted by DNA sonoporation, allowing for protein secretion into the ocular sphere. Sonoporation targeted to ciliary muscles has potential as a non-viral gene delivery procedure for the treatment of various ocular diseases.

\section{Conclusion}

UTMD has evolved as a promising method for non-invasive, target-specific gene delivery. The low toxicity and simplicity of in vivo application make this technology particularly attractive. The combination of UTMD and viral vectors in gene delivery not only enhanced the efficiency, but also abolished immunogenicity. UTMD is a promising technique for ocular gene delivery. However, the application of UTMD is still in its infancy stage and far from ready to be used in clinical applications. There remain certain unresolved issues. The ultrasound exposure parameters, frequency, mechanical index and amount of the plasmid DNA used should be optimized. The microbubble size and surface architecture should be optimized to prolong circulation time and gene-loading efficiency.

With regard to ocular gene therapy, most available studies only evaluated the ocular tissue structure damage after gene therapy, but the impact on vision has not been sufficiently investigated. Further evaluation of the impact on vision is of high importance, particularly in view of eventual clinical application. Further research is required prior to the clinical application of UTMD. In spite of several problems remaining to be solved, UTMD is a promising system for ocular gene delivery.

\section{Acknowledgements}

The present review was supported by grants from the Natural Science Foundation of Shanghai Science Commission (grant no. 11ZR1421100), the Scientific Effort Project of Shanghai Science and Technology committee (grant no. 1441968200) and the Natural Science Foundation of China (grant no. 81200700).

\section{References}

1. Surace EM and Auricchio A: Versatility of AAV vectors for retinal gene transfer. Vision Res 48: 353-359, 2008.

2. Zhang L, Li X, Zhao M, He P, Yu W, Dong J et al: Antisense oligonucleotide targeting c-fos mRNA limits retinal pigment epithelial cell proliferation; a key step in the progression of proliferative vitreoretinopathy. Exp Eye Res 83: 1405-1411, 2006.

3. Kuliszewski MA, Kobulnik J, Lindner JR, Stewart DJ and Leong-Poi H: Vascular gene transfer of SDF-1 promotes endothelial progenitor cell engraftment and enhances angiogenesis in ischemic muscle. Mol Ther 19: 895-902, 2011.

4. Li HL, Zheng XZ, Wang HP, Li F, Wu Y and Du LF: Ultrasound-targeted microbubble destruction enhances AAV-mediated gene transfection in human RPE cells in vitro and rat retina in vivo. Gene Ther 16: 1146-1153, 2009.

5. Zhigang W, Zhiyu L, Haitao R, et al: Ultrasound-mediated microbubble destruction enhances VEGF gene delivery to the infarcted myocardium in rats. Clin Imaging 28: 395-398, 2004.

6. Zhang Q, Wang Z, Ran H, et al: Enhanced gene delivery into skeletal muscles with ultrasound and microbubble techniques. Acad Radiol 13: 363-367, 2006.

7. Ren JL, Wang ZG, Zhang Y, et al: Transfection efficiency of TDL compound in HUVEC enhanced by ultrasound-targeted microbubble destruction. Ultrasound Med Biol 34: 1857-1867, 2008.

8. Chen S, Shimoda M, Chen J and Grayburn PA: Stimulation of adult resident cardiac progenitor cells by durable myocardial expression of thymosin beta 4 with ultrasound-targeted microbubble delivery. Gene Ther 20: 225-233, 2013.

9. Sonoda S, Tachibana K, Uchino E, et al: Gene transfer to corneal epithelium and keratocytes mediated by ultrasound with microbubbles. Invest Ophthalmol Vis Sci 47: 558-564, 2006.

10. Chen S, Shimoda M, Wang MY, et al: Regeneration of pancreatic islets in vivo by ultrasound-targeted gene therapy. Gene Ther 17: 1411-1420, 2010. 
11. Li HL, Zheng XZ, Wang HP, Li F, Wu Y and Du LF: Ultrasound-targeted microbubble destruction enhances $\mathrm{AAV}$-mediated gene transfection in human RPE cells in vitro and rat retina in vivo. Gene Ther 16: 1146-1153, 2009.

12. Xie W, Liu S, Su H, Wang Z, Zheng Y and Fu Y: Ultrasound microbubbles enhance recombinant adeno-associated virus vector delivery to retinal ganglion cells in vivo. Acad Radiol 17: 1242-1248, 2010.

13. Sonoda S, Tachibana K, Yamashita T, et al: Selective gene transfer to the retina using intravitreal ultrasound irradiation. J Ophthalmol 2012: 412752, 2012.

14. Hippert C, Ibanes S, Serratrice N, et al: Corneal transduction by intra-stromal injection of AAV vectors in vivo in the mouse and ex vivo in human explants. PLoS One 7: e35318, 2012.

15. Kamata Y, Okuyama T, Kosuga M, et al: Adenovirus-mediated gene therapy for corneal clouding in mice with mucopolysaccharidosis type VII. Mol Ther 4: 307-312, 2001.

16. Klausner EA, Peer D, Chapman RL, Multack RF and Andurkar SV: Corneal gene therapy. J Control Release 124: 107-133, 2007

17. Liu HA, Liu YL, Ma ZZ, Wang JC and Zhang Q: A lipid nanoparticle system improves siRNA efficacy in RPE cells and a laser-induced murine CNV model. Invest Ophthalmol Vis Sci 52: 4789-4794, 2011

18. Shafaa MW, El Shazly LH, El Shazly AH, El gohary AA El hossary GG: Efficacy of topically applied liposome-bound tetracycline in the treatment of dry eye model. Vet Ophthalmol 14 18-25, 2011.

19. Dalkara D, Kolstad KD, Caporale N, et al: Inner limiting membrane barriers to AAV-mediated retinal transduction from the vitreous. Mol Ther 17: 2096-2102, 2009.

20. Peyman GA, Lad EM and Moshfeghi DM: Intravitreal injection of therapeutic agents. Retina 29: 875-912, 2009.

21. Wu $\mathrm{H}$ and Chen TC: The effects of intravitreal ophthalmic medications on intraocular pressure. Semin Ophthalmol 24: 100-105, 2009.

22. Peeters L, Lentacker I, Vandenbroucke RE, et al: Can ultrasound solve the transport barrier of the neural retina? Pharm Res 25 2657-2665, 2008

23. Du J, Shi QS, Sun Y, et al: Enhanced delivery of monomethoxypoly(ethylene glycol)-poly(lactic-co-glycolic acid)-poly l-lysine nanoparticles loading platelet-derived growth factor BB small interfering RNA by ultrasound and/or microbubbles to rat retinal pigment epithelium cells. J Gene Med 13: 312-323, 2011.

24. Bishop P: The biochemical structure of mammalian vitreous. Eye (Lond) 10 (Pt 6): 664-670, 1996.

25. Peeters L, Sanders NN, Braeckmans K, et al: Vitreous: a barrier to nonviral ocular gene therapy. Invest Ophthalmol Vis Sci 46 : 3553-3561, 2005.

26. Nishikawa M and Huang L: Nonviral vectors in the new millennium: delivery barriers in gene transfer. Hum Gene Ther 12: 861-870, 2001

27. Manickan E, Smith JS, Tian J, et al: Rapid Kupffer cell death after intravenous injection of adenovirus vectors. Mol Ther 13: 108-117, 2006.

28. Igarashi T, Miyake N, Fujimoto C, et al: Adeno-associated virus type 8 vector-mediated expression of siRNA targeting vascular endothelial growth factor efficiently inhibits neovascularization in a murine choroidal neovascularization model. Mol Vis 20: 488-496, 2014.

29. Huang C, Cen LP, Liu L, et al: Adeno-associated virus-mediated expression of growth-associated protein- 43 aggravates retinal ganglion cell death in experimental chronic glaucomatous injury. Mol Vis 19: 1422-1432, 2013.

30. Ikeda Y, Yonemitsu Y, Miyazaki M, et al: Acute toxicity study of a simian immunodeficiency virus-based lentiviral vector for retinal gene transfer in nonhuman primates. Hum Gene Ther 20: 943-954, 2009.

31. Cavazzana-Calvo $\mathrm{M}$ and Fischer A: Gene therapy for severe combined immunodeficiency: are we there yet? J Clin Invest 117 1456-1465, 2007

32. Wang Z, Storb R, Lee D, et al: Immune responses to AAV in canine muscle monitored by cellular assays and noninvasive imaging. Mol Ther 18: 617-624, 2010.

33. Wilson JM: Lessons learned from the gene therapy trial for ornithine transcarbamylase deficiency. Mol Genet Metab 96: 151-157, 2009.

34. Wu J, Zhang S, Wu X, et al: Enhanced transduction and improved photoreceptor survival of retinal degeneration by the combinatorial use of rAAV2 with a lower dose of adenovirus. Vision Res 48: 1648-1654,2008.
35. Provost N, Le Meur G, Weber M, et al: Biodistribution of rAAV vectors following intraocular administration: evidence for the presence and persistence of vector DNA in the optic nerve and in the brain. Mol Ther 11: 275-283, 2005.

36. Park HJ, Yang F and Cho SW: Nonviral delivery of genetic medicine for therapeutic angiogenesis. Adv Drug Deliv Rev 64: 40-52, 2012.

37. Nayerossadat N, Maedeh T and Ali PA: Viral and nonviral delivery systems for gene delivery. Adv Biomed Res 1: 27, 2012.

38. Bloquel C, Bourges JL, Touchard E, Berdugo M, BenEzra D and Behar-Cohen F: Non-viral ocular gene therapy: potential ocular therapeutic avenues. Adv Drug Deliv Rev 58: 1224-1242, 2006.

39. Thrimawithana TR, Young S, Bunt CR, Green C and Alany RG Drug delivery to the posterior segment of the eye. Drug Discov Today 16: 270-277, 2011.

40. Gaudana R, Ananthula HK, Parenky A and Mitra AK: Ocular drug delivery. AAPS J 12: 348-360, 2010.

41. Jayaraman MS, Bharali DJ, Sudha T and Mousa SA: Nano chitosan peptide as a potential therapeutic carrier for retinal delivery to treat age-related macular degeneration. Mol Vis 18: 2300-2308, 2012.

42. Zhou H, Yang L, Li H, et al: Downregulation of VEGF mRNA expression by triamcinolone acetonide acetate-loaded chitosan derivative nanoparticles in human retinal pigment epithelial cells. Int J Nanomedicine 7: 4649-4660, 2012.

43. Bainbridge JW, Tan MH and Ali RR: Gene therapy progress and prospects: the eye. Gene Ther 13: 1191-1197, 2006.

44. Han S, Mahato RI, Sung YK and Kim SW: Development of biomaterials for gene therapy. Mol Ther 2: 302-317, 2000.

45. Yamashita T, Sonoda S, Suzuki R, et al: A novel bubble liposome and ultrasound-mediated gene transfer to ocular surface: RC-1 cells in vitro and conjunctiva in vivo. Exp Eye Res 85: 741-748, 2007.

46. Luo J, Zhou X, Diao L and Wang Z: Experimental research on wild-type p53 plasmid transfected into retinoblastoma cells and tissues using an ultrasound microbubble intensifier. J Int Med Res 38: 1005-1015, 2010.

47. Daigeler A, Chromik AM, Haendschke K, et al: Synergistic effects of sonoporation and taurolidin/TRAIL on apoptosis in human fibrosarcoma. Ultrasound Med Biol 36: 1893-1906, 2010

48. Lin CY, Liu TM, Chen CY, et al: Quantitative and qualitative investigation into the impact of focused ultrasound with microbubbles on the triggered release of nanoparticles from vasculature in mouse tumors. J Control Release 146: 291-298, 2010.

49. Wang Y, Zhou J, Zhang Y, Wang X and Chen J: Delivery of TFPI-2 using SonoVue and adenovirus results in the suppression of thrombosis and arterial re-stenosis. Exp Biol Med (Maywood) 235: 1072-1081, 2010.

50. Sirsi SR and Borden MA: Advances in ultrasound mediated gene therapy using microbubble contrast agents. Theranostics 2 : 1208-1222, 2012

51. Liang HD, Tang J and Halliwell M: Sonoporation, drug delivery and gene therapy. Proc Inst Mech Eng H 224: 343-361, 2010.

52. Lawrie A, Brisken AF, Francis SE, et al: Ultrasound enhances reporter gene expression after transfection of vascular cells in vitro. Circulation 99: 2617-2620, 1999.

53. Taniyama Y, Tachibana K, Hiraoka K, et al: Development of safe and efficient novel nonviral gene transfer using ultrasound: enhancement of transfection efficiency of naked plasmid DNA in skeletal muscle. Gene Ther 9: 372-380, 2002

54. Zhou Y, Yang K, Cui J, Ye JY and Deng CX: Controlled permeation of cell membrane by single bubble acoustic cavitation. J Control Release 157: 103-111, 2012

55. Chen H, Kreider W, Brayman AA, Bailey MR and Matula TJ: Blood vessel deformations on microsecond time scales by ultrasonic cavitation. Phys Rev Lett 106: 034301, 2011.

56. Hauser J, Ellisman M, Steinau HU, Stefan E, Dudda M and Hauser M: Ultrasound enhanced endocytotic activity of human fibroblasts. Ultrasound Med Biol 35: 2084-2092, 2009.

57. Li W, Liu S, Ren J, Xiong H, Yan X and Wang Z: Gene transfection to retinal ganglion cells mediated by ultrasound microbubbles in vitro. Acad Radiol 16: 1086-1094, 2009.

58. Zhou XY, Liao Q, Pu YM, et al: Ultrasound-mediated microbubble delivery of pigment epithelium-derived factor gene into retina inhibits choroidal neovascularization. Chin Med J (Engl) 122: 2711-2717, 2009.

59. Lee SY, Huh MS, Lee S, et al: Stability and cellular uptake of polymerized siRNA (poly-siRNA)/polyethylenimine (PEI) complexes for efficient gene silencing. J Control Release 141: 339-346, 2010 
60. Howard CM, Forsberg F, Minimo C, Liu JB, Merton DA and Claudio PP: Ultrasound guided site specific gene delivery system using adenoviral vectors and commercial ultrasound contrast agents. J Cell Physiol 209: 413-421, 2006.

61. Geers B, Lentacker I, Alonso A, et al: Elucidating the mechanisms behind sonoporation with adeno-associated virus-loaded microbubbles. Mol Pharm 8: 2244-2251, 2011.

62. Li F, Jin L, Wang H, et al: The dual effect of ultrasound-targeted microbubble destruction in mediating recombinant adeno-associated virus delivery in renal cell carcinoma: transfection enhancement and tumor inhibition. J Gene Med 16: 28-39, 2014.

63. Mueller $\mathrm{C}$ and Flotte TR: Clinical gene therapy using recombinant adeno-associated virus vectors. Gene Ther 15: 858-863, 2008

64. Zheng X, Du L, Wang H and Gu Q: A novel approach to attenuate proliferative vitreoretinopathy using ultrasound-targeted microbubble destruction and recombinant adeno-associated virus-mediated RNA interference targeting transforming growth factor-beta2 and platelet-derived growth factor-B. J Gene Med 14: 339-347, 2012.

65. Zheng XZ, Wu Y, Li HL, Du LF, Wang HP and Gu Q: Comparative analysis of the effects of ultrasound-targeted microbubble destruction on recombinant adeno-associated virusand plasmid-mediated transgene expression in human retinal pigment epithelium cells. Mol Med Rep 2: 937-942, 2009.

66. Müller OJ, Schinkel S, Kleinschmidt JA, Katus HA and Bekeredjian R: Augmentation of AAV-mediated cardiac gene transfer after systemic administration in adult rats. Gene Ther 15 1558-1565, 2008.

67. Jin LF, Li F, Wang HP, Wei F, Qin P and Du LF: Ultrasound targeted microbubble destruction stimulates cellular endocytosis in facilitation of adeno-associated virus delivery. Int $\mathbf{J}$ Mol Sci 14: 9737-9750, 2013.

68. van Wamel A, Kooiman K, Harteveld M, et al: Vibrating microbubbles poking individual cells: drug transfer into cells via sonoporation. J Control Release 112: 149-155, 2006.
69. Sorace AG, Warram JM, Umphrey $H$ and Hoyt K: Microbubble-mediated ultrasonic techniques for improved chemotherapeutic delivery in cancer. J Drug Target 20: 43-54, 2012.

70. Heath CH, Sorace A, Knowles J, Rosenthal E and Hoyt K: Microbubble therapy enhances anti-tumor properties of cisplatin and cetuximab in vitro and in vivo. Otolaryngol Head Neck Surg 146: 938-945, 2012.

71. Lee NG, Berry JL, Lee TC, et al: Sonoporation enhances chemotherapeutic efficacy in retinoblastoma cells in vitro. Invest Ophthalmol Vis Sci 52: 3868-3873, 2011.

72. Zheng MM, Zhou XY, Wang LP and Wang ZG: Experimental research of RB94 gene transfection into retinoblastoma cells using ultrasound-targeted microbubble destruction. Ultrasound Med Biol 38: 1058-1066, 2012.

73. Campbell M, Nguyen AT, Kiang AS, et al: An experimental platform for systemic drug delivery to the retina. Proc Natl Acad Sci USA 106: 17817-17822, 2009.

74. Baseri B, Choi JJ, Tung YS and Konofagou EE: Multi-modality safety assessment of blood-brain barrier opening using focused ultrasound and definity microbubbles: a short-term study. Ultrasound Med Biol 36: 1445-1459, 2010.

75. Liu HL, Hua MY, Chen PY, et al: Blood-brain barrier disruption with focused ultrasound enhances delivery of chemotherapeutic drugs for glioblastoma treatment. Radiology 255: 415-425, 2010.

76. Park J, Zhang Y, Vykhodtseva N, Akula JD and McDannold NJ: Targeted and reversible blood-retinal barrier disruption via focused ultrasound and microbubbles. PLoS One 7: e42754, 2012.

77. Sheikov N, McDannold N, Sharma S and Hynynen K: Effect of focused ultrasound applied with an ultrasound contrast agent on the tight junctional integrity of the brain microvascular endothelium. Ultrasound Med Biol 34: 1093-1104, 2008.

78. Kowalczuk L, Boudinet M, El Sanharawi M, et al: In vivo gene transfer into the ocular ciliary muscle mediated by ultrasound and microbubbles. Ultrasound Med Biol 37: 1814-1827, 2011. 